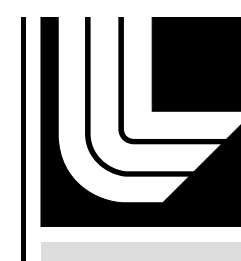

LAW RENCE LIVERMORE NATIO N A L LABORATORY

\title{
Design Aspects of an MSE Diagnostic for ITER
}

T.A. Casper, J. Jayakumar, M.A. Makowski, R. Ellis

April 19, 2004

Review of Scientific Instruments 
This document was prepared as an account of work sponsored by an agency of the United States Government. Neither the United States Government nor the University of California nor any of their employees, makes any warranty, express or implied, or assumes any legal liability or responsibility for the accuracy, completeness, or usefulness of any information, apparatus, product, or process disclosed, or represents that its use would not infringe privately owned rights. Reference herein to any specific commercial product, process, or service by trade name, trademark, manufacturer, or otherwise, does not necessarily constitute or imply its endorsement, recommendation, or favoring by the United States Government or the University of California. The views and opinions of authors expressed herein do not necessarily state or reflect those of the United States Government or the University of California, and shall not be used for advertising or product endorsement purposes. 


\title{
Design Aspects of an MSE Diagnostic for ITER
}

\author{
T.A. Casper, J. Jayakumar, M.A. Makowski, and R. Ellis \\ Lawrence Livermore National Laboratory, Livermore, California, USA
}

\begin{abstract}
The Motional Stark Effect (MSE) diagnostic is unique in its ability to measure the current profile and will be essential in ITER for detailed analysis of Advanced Tokamak (AT) and other types of discharges. However, design of a MSE diagnostic for ITER presents many unique challenges. Among these is optical analysis for the convoluted optical path, required for effective neutron shielding, that employs several reflective optics arranged to form a labyrinth. The geometry of the diagnostic has been laid out and the expected Doppler shifts and channel resolution calculated. A model of the optical train has also been developed based on the Mueller matrix formalism. Unfolding the pitch angle for this complicated geometry is not straightforward and possible methods are evaluated. The CORSICA code is used to model a variety of ITER discharges including start-up, Ipramp and reverse shear. The code also incorporates a synthetic MSE diagnostic that can be used to evaluate different viewing locations and optimize channel locations for the above discharges. Simulation of the optical emission spectrum is also underway.
\end{abstract}

\section{Introduction}

Operation of the ITER experiment requires instrumentation capable of surviving the high level of neutron fluence accumulated during the duration of operation to meet its design goals. It is likely that DD and DT operation will begin as early as possible to explore issues surrounding burning plasma operation. For this reason, the successful operation of instrumentation systems must be critically analyzed before installation and certainly prior to the intense neutron environment expected. We are beginning to explore issues associated with the measurement capabilities of several instrumentation systems to help understand and guarantee the utility of diagnostics and measurements throughout the life of ITER spanning several modes of operation. Particular challenges are expected to arise for advanced tokamak operations scenarios that may involve detailed measurement and control of current density profiles to achieved enhanced performance parameters and high- $\beta$ operation. It is anticipated that a variety of scenarios will include regions with high parameter gradients such as presently observed in discharges with internal transport barriers and in H-mode edge pedestals. On the DIII-D experiment, our MSE diagnostic [1] has been invaluable for determining the equilibrium in high- $\beta$ discharges, for accessing current-profile-controlled scenarios and, more recently, for observation and measurement of poloidal field fluctuations resulting from MHD activity [2]. In order to extrapolate to ITER operations, we are exploring these issues and capabilities of such an MSE diagnostic [3] via plasma simulation methods.

\section{CORSICA transport and diagnostic modeling}

We use the CORSICA code [4] to explore various operating scenarios for existing and future experiments. CORSICA is a predictive, time-dependent, 2D-equilibrium and 1D-transport code that has been used extensively to model and design DIII-D experiments [5,6]. It has also been used to explore operations scenarios for ITER , FIRE This work was performed under the auspices of the U.S. Department of Energy by the University of California, Lawrence Livermore National Laboratory under contract No. W-7405-Eng-48 
and KSTAR [7]. The equilibrium package [8] embedded in CORSICA was used extensively during design of the poloidal field system for ITER. This free-boundary equilibrium solver is integrated with the transport modeling capabilities within CORSICA to provide the capability for full discharge simulations involving timeevolution of the plasma shape, current density (magnetic field) and kinetic profiles. Both standard reference case and transport barrier scenarios for ITER have been simulated. We have recently added synthetic diagnostic capabilities that allow us to explore issues surrounding the ability of various measurement techniques to resolve plasma profiles in the presence of noisy measurements.

\section{MSE modeling}

We implemented an initial capability to simulate MSE data for these timeevolving discharge simulations. The design of and MSE instrument for ITER is being extensively studied and we have implemented a currently available description of such an instrument [3]. Simulations presented here use the geometry representative of an instrument viewing the heating neutral beam HNB4 from Port 3 [9]. We assume a $5 \mathrm{cmX} 5 \mathrm{~cm}$ rectangular area is viewed (spatial resolution) with a depth of view of $50 \mathrm{~cm}$ given approximately by the neutral beam width (a photon emission source from a $1250 \mathrm{cc}$ volume) with a distance from the source to the detector of $5 \mathrm{~m}$ to estimate the solid angle viewed. Currently we have included no polarization changes or losses that would result from the mirror labyrinth required on ITER. For data representative of an MSE measurement, we simulate quadrature detection of the sigma line component consistent with the measurement technique using PEMs on DIIID. This synthetic diagnostic technique provides the ability to explore operation of the instrument and its capability for resolving the current density using noisy measurements of the magnetic field pitch angle. Most diagnostic and neutral beam injection parameters are readily changed from the user interface either prior to or during a simulation or with post processor scripts. We can thus study MSE design issues such as the effects of various neutral beam and diagnostic geometry trade-offs that will be necessitated by the unique problems associated with the size of ITER and shielding from the neutron flux. These limitations, due to the nature of the ITER device, result in unavoidable compromises to the measurements possible. With the transport simulations coupled to the synthetic diagnostic, the consequences of these trade-offs can be explored with realistic data simulations.

\section{Simulation results}

Time-dependent simulations that explore shape evolution, X-point formation and flat-top burn conditions are run with the MSE diagnostic available. In these simulations, we employ a model for thermal transport based on the GLF23 [10] transport model in the core region with reduced transport at the plasma edge (where the GLF23 model is not yet applicable) to generate pedestal formation for H-mode-like conditions or to create an internal transport barrier. These simulations provide current density profile evolution consistent with this model for evolution of discharge parameters. For these simulations, except for the alpha particles, we have not included particle transport but rather prescribe an H-mode-like electron density profile. The alpha particle density is evolved using a rate equation to provide buildup plus a diffusion model to limit the of the alpha particle population. The neutral beam injection, specified for ITER [9] as two beam modules at 
$1 \mathrm{MeV}$ each providing $16.5 \mathrm{MW}$ of power injected tangentially to the major radius along the reference beam line, is provided by a Monte-Carlo deposition model [11] to give the buildup of beam densities, current drive and heating during plasma evolution. Interaction of beam particles with the background electrons provides the source of photons needed for an MSE measurement. Currently, we use a simple model for the source of photon flux by scaling the product of the neutral beam and electron densities consistent with the observed flux on DIII-D. A more detailed model will be incorporated in future work.

We show in Figure 1 time histories for an ITER simulation for an AT plasma with an internal transport barrier formed at about $130 \mathrm{~s}$ into the pulse representative of a $\mathrm{P}_{\text {fus }} \sim 170 \mathrm{MW}$ ITER discharge. We show in Figure 2 the detected pitch angles from our synthetic MSE instrument during the flattop burn conditions (350s) for this simulated discharge. We have simulated the noise consistent with photon statistics, namely noise proportional to the square root of the photon flux. We note that, for this particular viewing and neutral beam geometry, we obtain relatively good measurements of the pitch angle in the core region. Near the edge, however, for the beam injection parameters and plasma conditions simulated, the pitch angle measurements are consistently poor at larger radii. This is due to the low signal components resulting from the lack of beam density near the edge. Statistics associated with the ratio of noise-corrupted intensities used in the tangent function results in the noisy measurement characteristics at low signal strength. The quality of such measurements is obviously dependent on the plasma discharge conditions and both the neutral beam injection and MSE viewing geometries. We now have the capability to explore these issues in time-dependent-simulated plasmas with the intent of optimizing the information achieved from such an instrument on ITER.

In the future, we will be improving on the model for the source of MSE photons, adding the spectral characteristics and spatial averaging effects, and providing simulations of the detailed optical components. These simulations will be used to explore both optimization of the diagnostic operation and prediction of the current measurement capabilities for a variety of operations scenarios anticipated for ITER. We are currently developing the capability for utilizing CORSICA in plasma control system applications to allow us to explore the possibility for MSE-based current profile control applications for ITER.

\section{References}

[1] Rice, B.W., et al., Rev. Sci. Instrum. 66 (1995) 373-375.

[2] Jayakumar, R.J., et. al., this conference paper A15.

[3] Lotte, P., ITPA report, Padua, Italy, 2003.

[4] Casper, T.A., et al., Plasma Phys. Control. Fusion 45 (2003) 1-16.

[5] Doyle, E.J., et al., 19th IAEA Fusion Energy Conf., Lyon, France, 2002, EX/C3-2.

[6] Casper, T.A., et al., Proc. 30th EPS Conf. on Controlled Fusion and Plasma Physics,

St. Petersburg, Russia, 2002.

[7] Pearlstein, L.D., et al., Proc. 28th EPS Conf. on Controlled Fusion and Plasma

Physics, Madeira, Portugal, 2001.

[8] LoDestro, L.L. and Pearlstein, L.D., Phys. Plasmas 1 (1994) 90.

[9] ITER Technical Basis Report, GA0 FDR 1 02-07-13 R1.0, Section 2.5.

[10] Kinsey, J.E., et al., Phys. Ref. Lett. 86 (2001) 814.

[11] Goldston, R.J., et al., J. Comp. Physics 43 (1981) 61. 
Figure 1. ITER internal transport barrier simulation showing (a) time histories of the heating powers and (b) time variation of the separatricies with the MSE detector locations indicated.

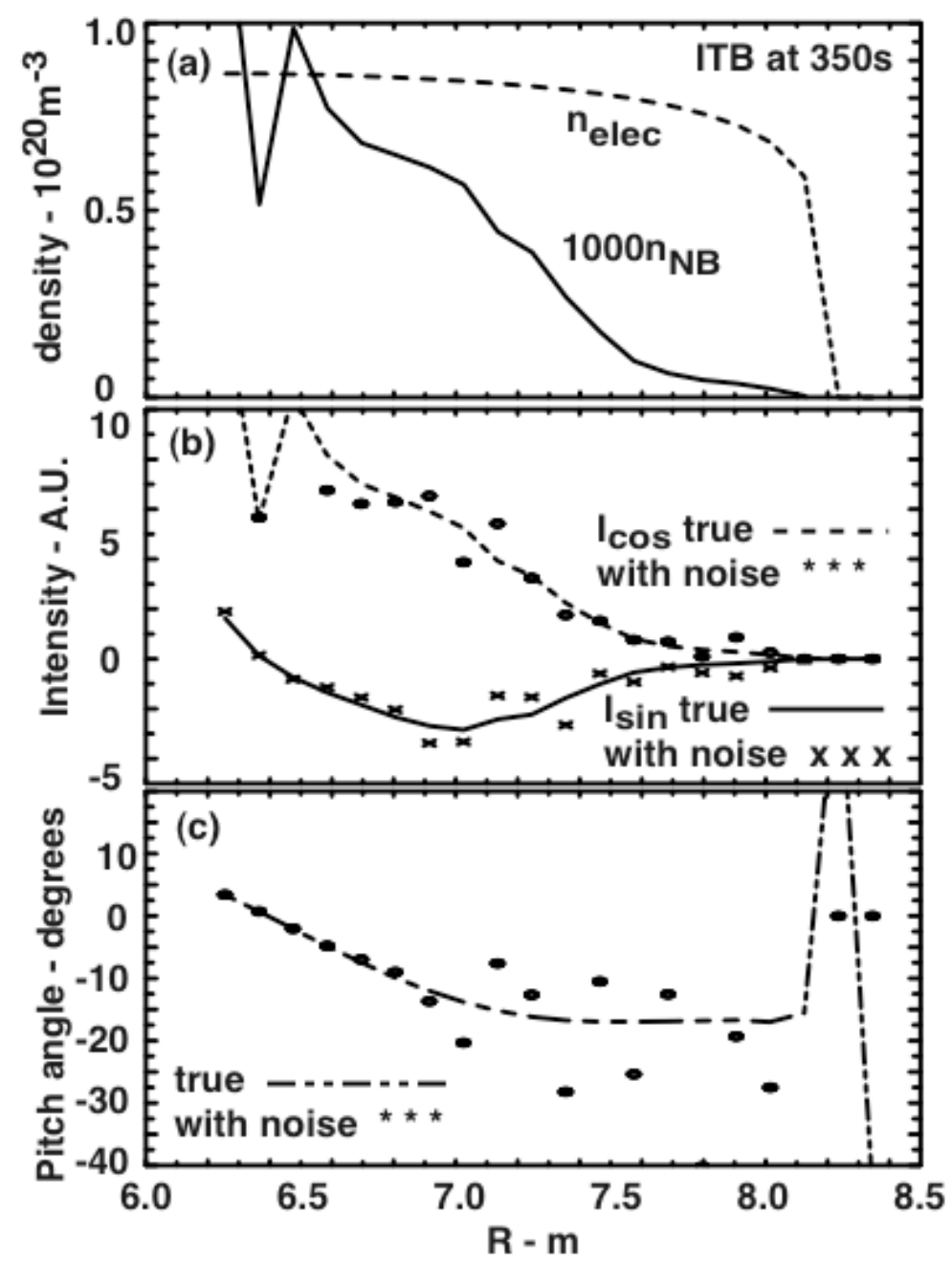

Figure 2. Simulation results at $\mathrm{t}=350 \mathrm{~s}$ for the ITB discharge simulation: (a) electron and beam densities, (b) simulated intensities detected of both signal and signal+noise, sin and cosine components and (c) true and measured pitch angles indicating good measurement capabilities in the core but poorer performance at the edge with low photon intensity . 